\title{
Necessary and Sufficient Conditions for Oscillations of Linear Delay Partial Difference Equations ${ }^{\text {th }}$
}

\author{
B.G. ZHANG ${ }^{\mathrm{a}, *}$ and S.T. LIU ${ }^{\mathrm{b}}$ \\ ${ }^{a}$ Department of Applied Mathematics, Ocean University of Qingdao, Qingdao 266003, China; ${ }^{\mathrm{b}}$ Department of Mathematics, \\ Binzhou Normal College, Binzhou, Shandong, 256604, China
}

(Received in final form 2 April 1997)

This paper is concerned with the linear delay partial difference equation

$$
A_{m, n}=\sum_{i=1}^{u} p_{i} A_{m-k_{i}, n-l_{i}}+\sum_{j=1}^{v} q_{j} A_{m+\tau_{j}, n+\sigma_{j}},
$$

where $p_{i}$ and $q_{j}$ are $r \times r$ matrices, $A_{m, n}=\left(a_{m, n}^{1}, a_{m, n}^{2}, \ldots, a_{m, n}^{r}\right)^{\mathrm{T}}, k_{i}, l_{i}, \tau_{j}$ and $\sigma_{j}$ are nonnegative integers, $u$ and $v$ are positive integers. Sufficient and necessary conditions for all solutions of this equation to be oscillatory componentwise are obtained.

Keywords: Partial difference equations, Oscillation, Characteristic equation

AMS Subject Classification: 39A10

\section{INTRODUCTION}

We consider the linear partial difference equation

$$
\begin{aligned}
A_{m, n} & =\sum_{i=1}^{u} p_{i} A_{m-k_{i}, n-l_{i}}+\sum_{j=1}^{v} q_{j} A_{m+\tau_{j}, n+\sigma_{j}}, \\
m, n & =0,1, \ldots,
\end{aligned}
$$

where $p_{i}$ and $q_{j}$ are $r \times r$ matrices, $A_{m, n}=\left(a_{m, n}^{1}\right.$, $\left.a_{m, n}^{2}, \ldots, a_{m, n}^{r}\right)^{\mathrm{T}}, k_{i}, l_{i}, \tau_{j}, \sigma_{j} \in N_{0}, i=1,2, \ldots, u, j=$ $1,2, \ldots, v, N_{t}=\{t, t+1, \ldots\}, u$ and $v$ are positive integers. By a solution of (1.1) we mean a sequence $\left\{A_{m, n}\right\}$ of $A_{m, n} \in R^{r}$, which satisfies (1.1) for $m, n \in N_{0}$.

A sequence of real numbers $\left\{a_{m, n}^{i}\right\}$ is said to oscillate if the terms $a_{m, n}^{i}$ are not all eventually positive or eventually negative. Let $\left\{A_{m, n}\right\}$ be a solution of (1.1) with $A_{m, n}=\left(a_{m, n}^{1}, \ldots, a_{m, n}^{r}\right)^{\mathrm{T}}$ for $m, n \in N_{0}$. We say that the solution $\left\{A_{m, n}\right\}$ oscillates componentwise if each component $\left\{a_{m, n}^{i}\right\}$ oscillates. Otherwise the solution $\left\{A_{m, n}\right\}$ is called nonoscillatory. Therefore a solution of (1.1) is

is The research was supported by NNSF of China.

* Corresponding author. 
nonoscillatory if it has a component $\left\{a_{m, n}^{i}\right\}$ which is eventually positive or eventually negative.

The solution $\left\{A_{m, n}\right\}$ of (1.1) is said to be proper, if there exist positive numbers $M, \alpha$ and $\beta$ such that

$$
\left\|A_{m, n}\right\| \leq M \alpha^{m} \beta^{n}, \quad \text { for } m, n \in N_{0} .
$$

In the following, we will show the sufficient conditions for all solutions to be proper for the linear difference system

$$
A_{m, n}=\sum_{i=1}^{u} p_{i} A_{m-k_{i}, n-l_{i}} .
$$

Set $k=\max k_{i}, l=\max l_{i}, i=1,2, \ldots, u, \Omega=N_{-k} \times$ $N_{-l} \backslash N_{0} \times N_{0}$. Given a function $\phi_{i, j}$ defined on $\Omega$, it is easy to construct by induction a double sequence $\left\{A_{i, j}\right\}$ which equals $\phi_{i, j}$ on $\Omega$ and satisfies (1.1) on $N_{0} \times N_{0}$. It is not difficult to prove that if the initial data $\phi_{m, n}$ satisfy (1.2) on $\Omega$, then the corresponding solution of (1.3) is proper. Similarly, we can find some conditions to guarantee that every solution of (1.1) to be proper.

Partial difference equations have been posed from various practical problems [3] and numerical analysis of partial differential equations [4].

Some sufficient conditions for all solutions of certain scalar partial difference equations to be oscillatory have been obtained in $[1,2,5-8]$.

The purpose of this paper is to derive a sufficient and necessary condition for all proper solutions of (1.1) to be oscillatory componentwise.

\section{SUFFICIENT AND NECESSARY CONDITIONS FOR OSCILLATIONS OF (1.1)}

THEOREM 2.1 Every proper solution $\left\{A_{m, n}\right\}$ of (1.1) oscillates componentwise if and only if its characteristic equation

$$
\operatorname{det}\left(\sum_{i=1}^{u} p_{i} \lambda^{-k_{i}} \mu^{-l_{i}}-I+\sum_{j=1}^{v} q_{j} \lambda^{\tau_{j}} \mu^{\sigma_{j}}\right)=0
$$

has no positive roots.
Proof The proof of "only if" is simple. Suppose to the contrary, let $\left(\lambda_{0}, \mu_{0}\right)$ be a positive root of (2.1), then there would be a nonzero vector $\zeta \in R^{r}$ such that

$$
\left(\sum_{i=1}^{u} p_{i} \lambda_{0}^{-k_{i}} \mu_{0}^{-l_{i}}-I+\sum_{j=1}^{\nu} q_{j} \lambda_{0}^{\tau_{j}} \mu_{0}^{\sigma_{j}}\right) \zeta=0,
$$

which implies that $A_{m, n}=\lambda_{0}^{m} \mu_{0}^{n} \zeta$ is a proper solution of (1.1) with at least one nonoscillatory component, which is a contradiction.

The proof of "if" uses the $z$-transform. Assume that (2.1) has no positive roots and (1.1) has a proper solution $\left\{A_{m, n}\right\}$ with at least one nonoscillatory component. Without loss of generality, we assume that $\left\{a_{m, n}^{1}\right\}$ is eventually positive. As (1.1) is autonomous, we may assume that $a_{m, n}^{1}>0$ for $m, n \in N_{0}$. For the proper solution $\left\{A_{m, n}\right\}$, the $z$-transform

$$
z\left(A_{m, n}\right)=\sum_{m, n=0}^{\infty} A_{m, n} z_{1}^{-m} z_{2}^{-n}=F\left(z_{1}, z_{2}\right)
$$

exists for $\left|z_{1}\right|>\alpha>0,\left|z_{2}\right|>\beta>0$. By taking the $z$-transform of both sides of (1.1) and using some formulas of the $z$-transform [6,7], we obtain

$$
\phi\left(z_{1}, z_{2}\right) F\left(z_{1}, z_{2}\right)=\psi\left(z_{1}, z_{2}\right),
$$

where

$$
\begin{aligned}
\phi\left(z_{1}, z_{2}\right)= & \sum_{i=1}^{u} p_{i} z_{1}^{-k_{i}} z_{2}^{-l_{i}}+\sum_{j=0}^{v} q_{j} z_{1}^{\tau_{j}} z_{2}^{\sigma_{j}}-I \\
\psi\left(z_{1}, z_{2}\right)=\sum_{j=1}^{v} q_{j} z_{1}^{\tau_{j}} z_{2}^{\sigma_{j}} & \sum_{m=0}^{\tau_{j}-1} \sum_{n=0}^{\infty} A_{m, n} z_{1}^{-m} z_{2}^{-n} \\
& +\sum_{n=0}^{\sigma_{j}-1} \sum_{m=0}^{\infty} A_{m, n} z_{1}^{-m} z_{2}^{-n} \\
& \left.-\sum_{m=0}^{\tau_{j}-1} \sum_{n=0}^{\sigma_{j}-1} A_{m, n} z_{1}^{-m} z_{2}^{-n}\right) .
\end{aligned}
$$

By the condition, $\operatorname{det} \phi\left(z_{1}, z_{2}\right) \neq 0$, for $z_{1} \times z_{2} \in$ $(0, \infty)^{2}$. Let $F_{1}\left(z_{1}, z_{2}\right)$ be the $z$-transform of the first component $\left\{a_{m, n}^{1}\right\}$ of the solution $\left\{A_{m, n}\right\}$ and let $b$ be the modulus of the largest zero of $\operatorname{det} \phi\left(z_{1}, z_{2}\right)$. 
Then by Cramer's rule, for $\left|z_{1}\right|>\max \{\alpha, b\},\left|z_{2}\right|>$ $\max \{\beta, b\}$,

$$
\operatorname{det} \phi\left(z_{1}, z_{2}\right) F_{1}\left(z_{1}, z_{2}\right)=\operatorname{det} D\left(z_{1}, z_{2}\right),
$$

where $D\left(z_{1}, z_{2}\right)$ has components of $\phi\left(z_{1}, z_{2}\right)$ and $\psi\left(z_{1}, z_{2}\right)$ as its entries and

$$
F_{1}\left(z_{1}, z_{2}\right)=\sum_{m, n=0}^{\infty} a_{m, n}^{1} z_{1}^{-m} z_{2}^{-n}
$$

Let

$$
w_{1}\left(z_{1}, z_{2}\right)=F_{1}\left(\frac{1}{z_{1}}, \frac{1}{z_{2}}\right)=\sum_{m, n=0}^{\infty} a_{m, n}^{1} z_{1}^{m} z_{2}^{n},
$$

which is a power series with positive coefficients having radius of convergence, $\rho_{i}, i=1,2$. Hence

$$
\operatorname{det} \phi\left(\frac{1}{z_{1}}, \frac{1}{z_{2}}\right) w_{1}\left(z_{1}, z_{2}\right)=\operatorname{det} D\left(\frac{1}{z_{1}}, \frac{1}{z_{2}}\right)
$$

for $\left|z_{i}\right|<\rho_{i}, i=1,2$. It is known that a power series with positive coefficients having radius of convergence $\rho_{i}, i=1,2$, has a singularity at $z_{i}=\rho_{i}, i=1,2$. By condition $\phi\left(z_{1}, z_{2}\right) \neq 0$, for $\left(z_{1}, z_{2}\right) \in(0, \infty) \times$ $(0, \infty)$. Thus $\phi\left(1 / \rho_{1}, 1 / \rho_{2}\right) \neq 0$, and hence

$$
w_{1}\left(z_{1}, z_{2}\right)=\frac{\psi\left(1 / z_{1}, 1 / z_{2}\right)}{\phi\left(1 / z_{1}, 1 / z_{2}\right)}
$$

is analytic in the region $\left|z_{1}-\rho_{1}\right|<d_{1}$ and $\left|z_{2}-\rho_{2}\right|<$ $d_{2}$, where $d_{1}$ and $d_{2}$ are positive, which contradicts the singularity of $w_{1}\left(z_{1}, z_{2}\right)$ at $z_{i}=\rho_{i}, i=1,2$. Therefore we must have $\rho_{i}=\infty, i=1$,2; i.e. (2.4) holds for $\left|z_{i}\right|>0, i=1,2$, which leads to $a_{m, n}^{1}=0$ for all large $m$ and $n$. Otherwise, for any fixed large numbers $M$ and $N$, the left-hand side of (2.4) has the nonzero term $b_{m, n} z_{1}^{-m} z_{2}^{-n}$, where $m \geq M$ and $n \geq N$. But the right-hand side of (2.4) has no such term. This contradiction proves Theorem 2.1.

For the scalar linear difference equation

$$
a_{m, n}=\sum_{i=1}^{u} p_{i} a_{m-k_{i}, n-l_{i}}+\sum_{j=1}^{j} q_{j} a_{m+\tau_{j}, n+\sigma_{j}},
$$

we have the following result.
COROLlaRY 2.1 Every proper solution of (2.6) oscillates if and only if the characteristic equation

$$
1=\sum_{i=1}^{u} p_{i} \lambda^{-k_{i}} \mu^{-l_{i}}+\sum_{j=1}^{v} q_{j} \lambda^{\tau_{j}} \mu^{\sigma_{j}}
$$

has no positive roots.

From Corollary 2.1 we can derive explicit conditions for every solution of some special equations to be oscillatory.

Consider the linear difference equation

$$
\begin{gathered}
q_{1} A_{m+1, n}+q_{2} A_{m, n+1}-p A_{m, n} \\
+\sum_{i=1}^{u} p_{i} A_{m-k_{i}, n-l_{i}}=0
\end{gathered}
$$

where $q_{1}, q_{2}, p$ and $p_{i}, i=1,2, \ldots, u$, are positive. The characteristic equation of (2.8) is

$$
\phi(\lambda, \mu)=q_{1} \lambda+q_{2} \mu-p+\sum_{i=1}^{u} p_{i} \lambda^{-k_{i}} \mu^{-l_{i}}=0 .
$$

COROLlary 2.2 Every proper solution of (2.8) is oscillatory, if

$$
\sum_{i=1}^{u} p_{i} \frac{\left(l_{i}+k_{i}+1\right)^{l_{i}+k_{i}+1} q_{1}^{k_{i}} q_{2}^{l_{i}}}{p^{l_{i}+k_{i}+1} k_{i}^{k_{i}} l_{i}^{l_{i}}}>1 .
$$

Proof By Corollary 2.1, it is sufficient to prove that under condition (2.10) the characteristic equation (2.9) has no positive roots. It is obvious that if $q_{1} \lambda+q_{2} \mu-p \geq 0$, then (2.9) has no positive roots. For the case that $q_{1} \lambda+q_{2} \mu-p<0$, we write $\phi(\lambda, \mu)$ in the form

$$
\begin{aligned}
\phi(\lambda, \mu)= & \left(p-q_{1} \lambda-q_{2} \mu\right) \\
& \times\left(-1+\sum_{i=1}^{u} p_{i} \frac{\lambda^{-k_{i}} \mu^{-l_{i}}}{p-q_{1} \lambda-q_{2} \mu}\right) .
\end{aligned}
$$

Set

$$
f(\lambda, \mu)=\frac{\lambda^{-k_{i}} \mu^{-l_{i}}}{p-q_{1} \lambda-q_{2} \mu} .
$$


It is easy to find that $f(\lambda, \mu)$ reaches its minimum value at

$$
\lambda_{0}=\frac{p k_{i}}{\left(k_{i}+l_{i}+1\right) q_{1}}, \quad \mu_{0}=\frac{p l_{i}}{\left(k_{i}+l_{i}+1\right) q_{2}} .
$$

Hence,

$$
\min _{0<q_{1} \lambda+q_{2} \mu<p} f(\lambda, \mu)=\frac{\left(k_{i}+l_{i}+1\right)^{k_{i}+l_{i}+1} q_{1}^{k_{i}} q_{2}^{l_{i}}}{p^{l_{i}+k_{i}+1} k_{i}^{k_{i}} l_{i}^{l_{i}}}
$$

Hence, for $0<q_{1} \lambda+q_{2} \mu<p$, we have

$$
\begin{aligned}
& \phi(\lambda, \mu) \geq\left(p-q_{1} \lambda-q_{2} \mu\right) \\
& \quad \times\left(-1+\sum_{i=1}^{u} p_{i} \frac{\left(k_{i}+l_{i}+1\right)^{k_{i}+l_{i}+1} q_{1}^{k_{i}} q_{2}^{l_{i}}}{p^{l_{i}+k_{i}+1} k_{i}^{k_{i}} l_{i}^{l_{i}}}\right)>0,
\end{aligned}
$$

which implies that (2.9) has no positive roots. By Corollary 2.1, every solution of (2.8) oscillates.

For $u=1,(2.10)$ is not only sufficient but also necessary for every solution of $(2.8)$ to be oscillatory.

\section{COROLlary 2.3 Every solution of the equation}

$$
q_{1} A_{m+1, n}+q_{2} A_{m, n+1}-p A_{m, n}+p_{1} A_{m-k, n-l}=0
$$

oscillates if and only if

$$
p_{1} \frac{(k+l+1)^{k+l+1} q_{1}^{k} q_{2}^{l}}{p^{l+k+1} k^{k} l^{l}}>1 .
$$

Proof To prove this theorem it is sufficient to prove that if (2.12) does not hold, then (2.11) has a positive solution. In fact, the characteristic equation of (2.11) is

$$
\phi(\lambda, \mu)=q_{1} \lambda+q_{2} \mu-p+p_{1} \lambda^{-k} \mu^{-l}=0 .
$$

Obviously,

$$
\phi\left(\frac{p(k+1)}{(k+l+1) q_{1}}, \frac{p l}{(k+l+1) q_{2}}\right)>0
$$

and

$$
\begin{aligned}
& \phi\left(\frac{p k}{(k+l+1) q_{1}}, \frac{p l}{(k+l+1) q_{2}}\right) \\
& \quad=\frac{p}{k+l+1}\left(-1+p_{1} \frac{(k+l+1)^{k+l+1} q_{1}^{k} q_{2}^{l}}{p^{l+k+1} k^{k} l^{l}}\right) \leq 0 .
\end{aligned}
$$

Since $\phi(\lambda, \mu)$ is continuous, then there exist

$$
\lambda_{0} \in\left(\frac{p k}{(k+l+1) q_{1}}, \frac{p(k+1)}{(k+l+1) q_{1}}\right)
$$

and

$$
\mu_{0}=\frac{p l}{(k+l+1) q_{2}}
$$

such that $\phi\left(\lambda_{0}, \mu_{0}\right)=0$. By Corollary 2.1, (2.11) has a positive solution. The proof is complete.

Remark 2.1 Corollaries 2.2 and 2.3 include the main results in $[6,7]$.

\section{References}

[1] S.S. Cheng and B.G. Zhang, Qualitative theory of partial difference equations (I): Oscillation of nonlinear partial difference equations, Tamkang J. Math. 25(3) (1994) 279288.

[2] B.G. Zhang, S.T. Liu and S.S. Cheng, Oscillation of a class of delay partial difference equations. J. Difference Equations and its Applications 1 (1995) 215-226.

[3] Li Xiang-ping, Partial difference equations used in the study of molecular orbits. Acta Chimica SINICA 40(8) (1982) 688-698.

[4] W.G. Kelley and A.C. Peterson, Difference Equations, Academic Press, New York, 1991.

[5] B.G. Zhang and S.T. Liu, Oscillations of partial difference equations. PanAmerican Math. J. 5 (1995) 61-70.

[6] B.G. Zhang and S.T. Liu, Necessary and sufficient conditions for oscillations of delay partial difference equations, Discussiones Mathematicae Differential Inclussions 15 (1995) 213-219.

[7] B.G. Zhang and S.T. Liu, Necessary and sufficient conditions for oscillations of partial difference equations, Nonlinear Studies 3(2) (1996) 187-192.

[8] B.G. Zhang and S.T. Liu, On the oscillation of two partial difference equations, J. Math. Anal. Appl. 206 (1997) 480492. 


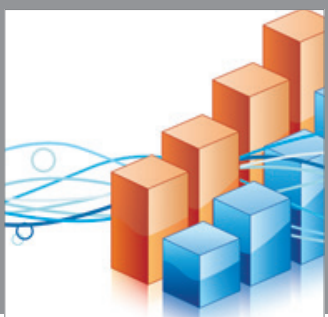

Advances in

Operations Research

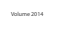

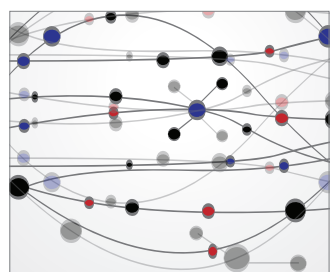

\section{The Scientific} World Journal
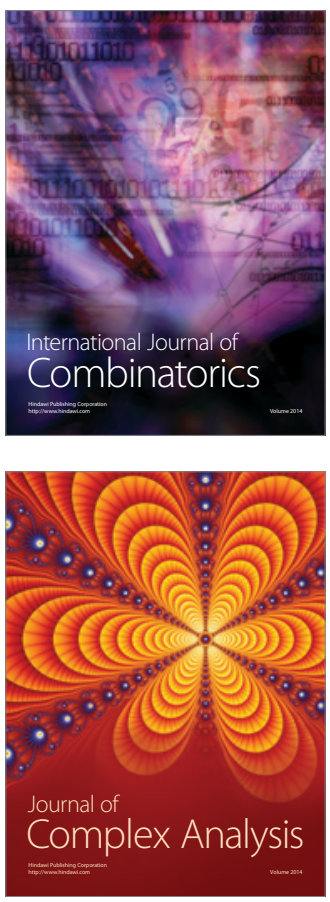

International Journal of

Mathematics and

Mathematical

Sciences
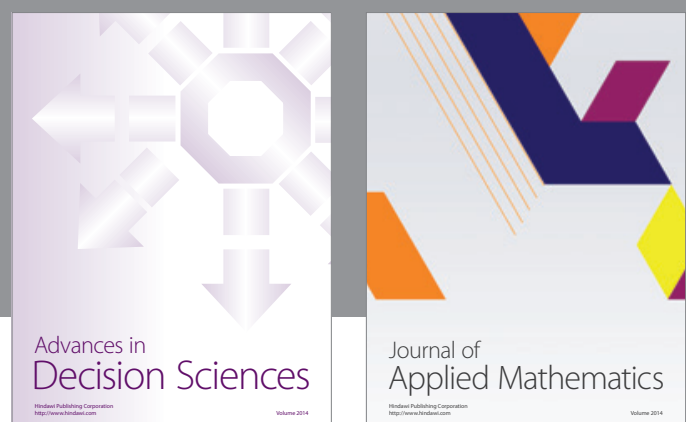

Journal of

Applied Mathematics
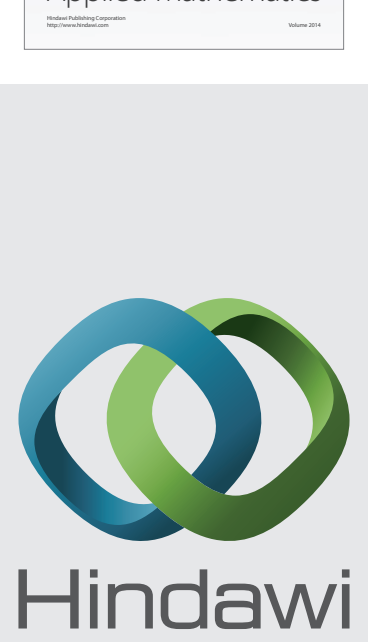

Submit your manuscripts at http://www.hindawi.com
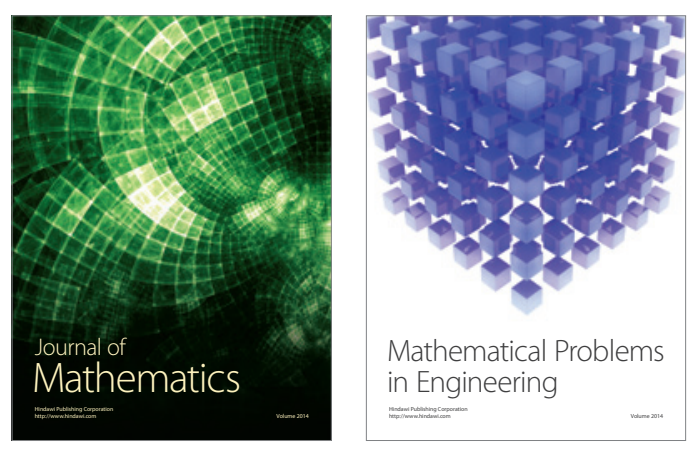

Mathematical Problems in Engineering
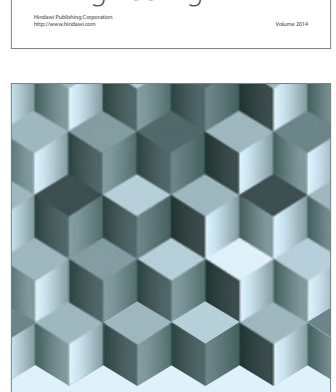

Journal of

Function Spaces
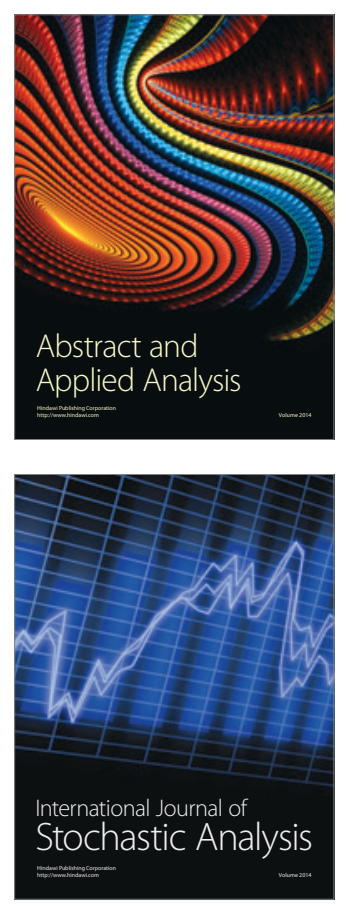

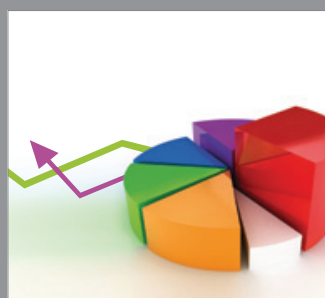

ournal of

Probability and Statistics

Promensencen
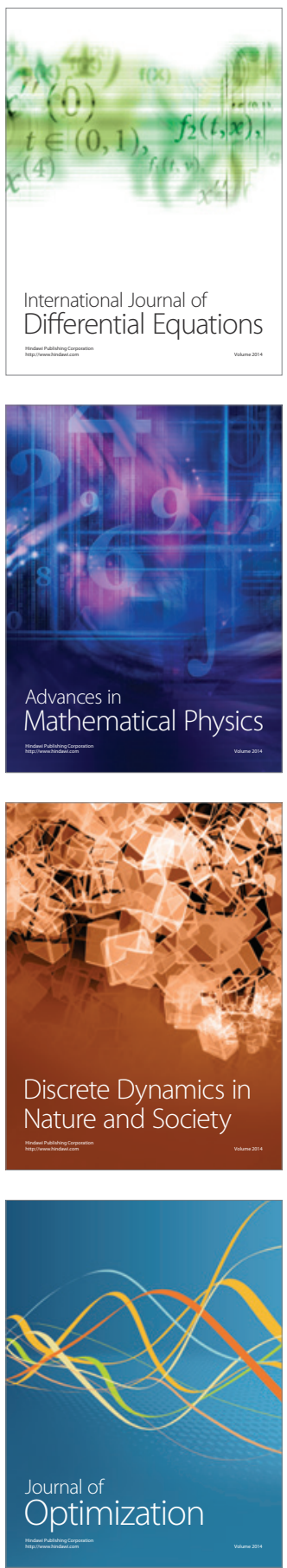